\title{
Sequential high-content profiling of the lgG-autoantibody repertoire reveals novel antigens in rheumatoid arthritis
}

\author{
Stefan Vordenbäumen ${ }^{1 *}$, Angelika Lueking ${ }^{2}$, Petra Budde ${ }^{2}$, Hans-Dieter Zucht ${ }^{2}$, Heike Goehler ${ }^{2}$, Ralph Brinks ${ }^{1,3}$, \\ Rebecca Fischer-Betz ${ }^{1}$, Jutta Richter ${ }^{1}$, Ellen Bleck' ${ }^{1}$ Jacqueline Detert ${ }^{4}$, Hans-Eckhard Langer ${ }^{5}$, Anne Sörgel ${ }^{1}$, \\ Gerd-Rüdiger Burmester ${ }^{4}$, Peter Schulz-Knappe ${ }^{2}$ and Matthias Schneider ${ }^{1}$
}

\begin{abstract}
Background: The aim was to identify novel diagnostic autoantibody candidates for rheumatoid arthritis (RA) by comprehensive screening for autoreactivity.

Method: We incubated 5892 recombinant proteins coupled to fluorescent beads, with patients' sera for the detection of IgG-autoantibodies in three independent patient cohorts: $A(n=72$ patients with established RA); B/B- $(n=116$ patients with early RA (B) and $n=51$ CCP-negative patients with early RA from $B(B-))$; and C ( $n=184$ patients with early seronegative RA), in comparison to matched healthy controls. Intersects of significantly increased autoantibodies as determined by the Mann-Whitney test were sought.

Result: Screening of 5892 antigens in RA cohorts A and B, or the seronegative cohorts B- and C revealed intersects of 23 and 13 significantly increased autoantibodies, respectively. Reactivity to three antigens was increased in all cohorts tested: N-acetylglucosamine-1-phosphate transferase, gamma subunit (GNPTG), heterogeneous nuclear ribonucleoprotein A1-like 2 (HNRNPA1), and insulin-like growth factor binding protein 2 (IGFBP2).
\end{abstract}

Conclusions: Comprehensive sequential screening for autoantibodies reveals novel candidates for diagnostic markers in both seropositive and seronegative RA and suggests new fields of research into the pathogenesis of RA.

Keywords: Rheumatoid arthritis, Autoantibody, Biomarker

\section{Background}

Rheumatoid arthritis (RA) is a chronic inflammatory autoimmune disease characterized by destruction of cartilage and bone. RA pathogenesis features numerous autoimmune processes such as autoreactive T cells and the formation of autoantibodies [1]. Amongst the autoantibodies present in RA sera, rheumatoid factor (RF) and antibodies against citrullinated peptides (ACPA) have emerged as important diagnostic and prognostic markers [2], although a range of other autoantibodies have been identified [3]. Evidence is accumulating that RF and ACPA are directly involved in the pathogenesis of the disease [4], potentially even prior to the occurrence of synovial inflammation [5].

\footnotetext{
* Correspondence: stefan.vordenbaeumen@med.uni-duesseldorf.de 'Department Rheumatology \& Hiller Research Unit Rheumatology, Heinrich-Heine-University, Moorenstr. 5, Düsseldorf 40225, Germany Full list of author information is available at the end of the article
}

Despite progress in the serologic diagnosis of RA introduced by the detection of these antibodies, a considerable number of patients with RA do not display RF and/or ACPA [6, 7]. In these cases, the diagnosis of RA relies heavily upon clinical presentation and imaging procedures. Thus, identification of reliable additional markers to recognize RF/ACPA-negative RA and to further ascertain the diagnosis of RF/ACPA-positive RA is desirable.

We adopted a multiplex bead-based approach to sequentially screen sera from three independent patient cohorts: cohort A, patients with established RA; cohort B, patients with early $\mathrm{RA}$; and cohort $\mathrm{C}$, patients with seronegative RA) for the presence of autoantibodies to over 5800 human antigens, aiming to (1) retrieve a collection of candidates for future diagnostic assay development, and (2) gain new insights into the pathogenesis of RA. 


\section{Methods}

Sample cohorts and autoantibody identification strategy In order to identify potential novel diagnostic autoantibodies, antibody profiles of serum samples from different RA patient cohorts were sequentially compared to healthy controls. Healthy controls were chosen in an age-adjusted and sex-adjusted manner from specimens from blood donors of the Bavarian Red Cross, Germany or collected from healthy individuals after the exclusion of rheumatic diseases from a community screening program. Patient cohorts were as follows. Cohort A (established RA) comprised 72 consecutive patients with established RA according to American College of Rheumatology (ACR)/ European League Against Rheumatism (EULAR) 2010 criteria (age $56.1 \pm 13.3$ years, $73.6 \%$ female, Disease Activity Score for 28 joints (DAS28) $3.5 \pm 2.3$, therapy: methotrexate $40 \%$, leflunomide $12.5 \%$, tumor necrosis factor alpha (TNFa)-blockade $18 \%$ ) from the outpatient department of Heinrich-Heine-University Düsseldorf. These patients were compared to 71 age-matched and sex-matched healthy controls (age 54.6 \pm 11.3 years, $73.2 \%$ female). Cohort B (early RA) comprised 116 patients with early RA from the HIT HARD study [8] (age 49.8 \pm 13.8 years, $71.3 \%$ female, DAS28 $6.1 \pm 1.0$, all therapy-naive). These patients were compared to 116 healthy controls (age $49.8 \pm 12.8$ years, $71.6 \%$ female). A subgroup analysis was conducted in seronegative patients from cohort B, termed B- $(\mathrm{n}=51$, age $54.5 \pm 13.3$ years, $66 \%$ female $)$. Cohort C (seronegative cohort) comprised 184 patients with ACPA-negative RA according to 2010 ACR/EULAR criteria (age $60.2 \pm 13.8$ years, $62.5 \% \%$ female, all therapynaive). These patients were compared to 184 healthy controls (age $55.2 \pm 10$ years, $62.5 \%$ female). All serum samples were obtained by standard procedures and stored at $-80{ }^{\circ} \mathrm{C}$ until use.

\section{Multiplex bead-based autoantibody detection}

We produced 5892 recombinant antigens in Escherichia coli and purified them. Five cDNA libraries originating from different human tissues (fetal brain, colon, lung, liver, Cd4 induced and non-induced T cells) were used for the recombinant production of human antigens. All of these cDNA libraries were oligo(dT)-primed, containing the coding region for an N-terminally located hexahistidine-tag and were under transcriptional control of the lactose inducible promoter from E. coli [9]. Sequence integrity of the cDNA libraries was confirmed by 5' DNA sequencing. Additionally, expression clones representing the full-length sequence derived from the human ORFeome collection [10] were included. Individual antigens were designed in silico, synthesized chemically (Life Technologies, Carlsbad, USA) and cloned into the expression vector $\mathrm{PQE30-NST}$ fused to the coding region for the N-terminal-located His6-tag. Of the antigens,
$73 \%$ were produced by cDNA library expression clones, $24 \%$ of the antigens derive from clones of the human ORFeome collection and $3 \%$ of the antigens were based on in silico design.

Recombinant gene expression was performed in $E$. coli SCS1 cells carrying plasmid pSE111 for improved expression of human genes [11]. Cells were cultivated in $200 \mathrm{ml}$ auto-induction medium (Overnight Express autoinduction medium, Merck, Darmstadt, Germany) overnight and harvested by centrifugation. Bacterial pellets were lysed by resuspension in $15 \mathrm{ml}$ lysis buffer (6 M guanidinium$\mathrm{HCl}, 0.1 \mathrm{M} \mathrm{NaH}_{2} \mathrm{PO}_{4}, 0.01 \mathrm{M}$ Tris- $\mathrm{HCl}, \mathrm{pH}$ 8.0). Soluble proteins were affinity-purified after binding to Protino ${ }^{\circ}$ Ni-IDA 1000 Funnel Column (Macherey-Nagel, Düren, Germany). Columns were washed with $8 \mathrm{ml}$ washing buffer (8 M urea, $0.1 \mathrm{M} \mathrm{NaH} \mathrm{PO}_{4}, 0.01 \mathrm{M}$ Tris- $\mathrm{HCl}$, $\mathrm{pH}$ 6.3). Proteins were eluted in $3 \mathrm{ml}$ elution buffer (6 M urea, $0.1 \mathrm{M} \mathrm{NaH}_{2} \mathrm{PO}_{4}, 0.01 \mathrm{M}$ Tris- $\mathrm{HCl}, 0.5 \%$ $(\mathrm{w} / \mathrm{v})$ trehalose $\mathrm{pH} 4.5)$. Each protein preparation was transferred into 2D-barcoded tubes, lyophilized and stored at $-20{ }^{\circ} \mathrm{C}$.

In this study, twenty different bead-based arrays containing up to 384 different proteins were used. For production of bead-based arrays (BBA), the proteins were coupled to magnetic carboxylated color-coded beads (MagPlex ${ }^{\mathrm{TM}}$ microspheres, Luminex Corporation, Austin, TX, USA). The manufacturer's protocol for coupling proteins to MagPlex $^{\mathrm{TM}}$ microspheres was adapted to use liquid handling systems. A semi-automated coupling procedure of one BBA encompassed 384 single, separate coupling reactions, which were carried out in four 96-well plates. For each single coupling reaction, up to $12.5 \mu \mathrm{g}$ antigen and $8.8 \times 10^{5}$ MagPlex $^{\mathrm{TM}}$ beads of one color region (ID) were used. All liquid handling steps were carried out by either an eight-channel pipetting system (Starlet, Hamilton Robotics, Bonaduz, Switzerland) or a 96-channel pipetting system (Evo Freedom 150, Tecan, Männderdorf, Switzerland). For semi-automated coupling, antigens were dissolved in $\mathrm{H}_{2} \mathrm{O}$, and aliquots of 60 microliters were transferred from 2D barcode tubes to 96-well plates. MagPlex ${ }^{\mathrm{TM}}$ microspheres were homogeneously resuspended and each bead ID was transferred in one well of a 96-well plate. The 96-well plates containing the microspheres were placed on a magnetic separator (LifeSep $^{\mathrm{Tm}}$, Dexter Magnetic Technologies Inc., Elk Grove Village, USA) to sediment the beads for washing steps and on a microtiter plate shaker (MTS2/4, IKA) to facilitate permanent mixing for incubation steps.

For coupling, the microspheres were washed three times with activation buffer $\left(100 \mathrm{mM} \mathrm{NaH} \mathrm{PO}_{4}, \mathrm{pH}\right.$ 6.2) and resuspended in $120 \mu \mathrm{l}$ activation buffer. To obtain reactive sulfo-NHS-ester intermediates, $15 \mu \mathrm{l}$ 1-ethly-3(3-dimethlyaminopropyl) carbodiimide $(50 \mathrm{mg} / \mathrm{ml})$ and $15 \mu \mathrm{N}$-hydroxy-succinimide $(50 \mathrm{mg} / \mathrm{ml})$ were applied 
to microspheres. After 20 minutes incubation $(900 \mathrm{rpm}$, room temperature (RT)) the microspheres were washed three times with coupling buffer (50 mM MES, pH 5.0) and resuspended in $65 \mu \mathrm{l}$ coupling buffer. Immediately, $60 \mu \mathrm{l}$ antigen solution was added to reactive microspheres and coupling took place over 120 minutes under permanent mixing (900 rpm, RT). After three wash cycles using washing buffer (PBS, $0.1 \%$ Tween20) coupled beads were resuspended in blocking buffer (PBS, $1 \%$ BSA, $0.05 \%$ ProClin300), incubated for 20 minutes (900 rpm, RT) and then transferred to be maintained at $4-8{ }^{\circ} \mathrm{C}$ for $12-72 \mathrm{~h}$.

To monitor the assay performance various proteins were used as control proteins and coupled individually to microspheres as described for antigens. Human and mouse IgG (Sigma-Aldrich, St. Louis, USA) were used to control the reactivity of the detection antibodies, BSA (Sigma-Aldrich) to monitor the background and E. coli lysate to detect serum antibodies directed against $E$. coli proteins. For coupling reaction control $10 \mu \mathrm{g}, 25 \mu \mathrm{g}$, $100 \mu \mathrm{g}$ and $200 \mu \mathrm{g}$ human IgG, $200 \mu \mathrm{g}$ mouse IgG, $1 \mu \mathrm{g}$ $\mathrm{BSA}$ and $500 \mu \mathrm{g} E$. coli proteins were used, respectively. A bead mix of a multiplex BBA was generated by pooling 384 antigen-coupled beads together with control proteincoupled beads. The BBA was stored at $4-8{ }^{\circ} \mathrm{C}$ until use.

Serum samples were transferred to 2D barcode tubes and a 1:100 serum dilution was prepared with assay buffer (PBS, $0.5 \%$ BSA, $10 \%$ E. coli lysate, 50 \% Low-Cross buffer (Candor Technologies, Nürnberg, Germany)) in 96-well plates. The serum dilutions were first incubated for 20 minutes to neutralize any human IgG eventually directed against $E$. coli proteins. The BBA was sonicated for 5 minutes and the bead mix was distributed in 96-well plates. After three wash cycles with washing buffer (PBS, $0.05 \%$ Tween20) serum dilutions $(50 \mu \mathrm{l})$ were added to the bead mix and incubated for $20 \mathrm{~h}$ (900 rpm, $\left.4-8{ }^{\circ} \mathrm{C}\right)$. Supernatants were removed from the beads by three wash cycles, and secondary R-phycoerythrinlabeled antibody $(5 \mu \mathrm{g} / \mathrm{ml}$, goat anti-human, Dianova, Hamburg, Germany) was added for a final incubation of 45 minutes ( $900 \mathrm{rpm}, \mathrm{RT}$ ). The beads were washed three times with washing buffer (PBS, $0.1 \%$ Tween20) and resuspended in $100 \mu \mathrm{l}$ sheath fluid (Luminex Corporation). Subsequently, beads were analyzed in a FlexMap3D device for fluorescent signal readout (DD gate 7.500-15.000; sample size: $80 \mu \mathrm{l}$; 1000 events per bead ID; timeout $60 \mathrm{sec}$ ). The binding events were displayed as median fluorescence intensity (MFI). Measurements were disregarded when low numbers of bead events ( $<30$ beads) were counted per bead ID.

\section{Statistical analysis}

Our antigen prioritization approach was sequential performance of the Mann-Whitney test to identify intersects of significantly upregulated markers in the patient cohorts compared to matched healthy controls. For data preprocessing and normalization, if fewer than 30 beads were counted for a specific antigen, the corresponding MFI value was set to missing. Samples or antigens were discarded from further analysis if either a patient sample or an antigen had $>20 \%$ missing values. In total, six patients and $1.5 \%$ of antigens tested were discarded because of $>20 \%$ missing values. Prior to normalization, MFI values were log2-transformed. Quantile normalization was used to normalize data on each individual plate by BBA according to [12]. A missing MFI value for an antigen was replaced by the respective median value across all samples for this specific antigen. Replacement of the data by median imputed values was necessary for $<0.25 \%$ without notable accumulation in certain antigens. The target variable for all statistical work was the MFI of the detection antibody.

Antigens with an upregulated MFI and fold change $\geq 1$ were further considered. The fold change was calculated as the ratio of median MFI of cases vs. controls. In each cohort, receiver operating characteristic (ROC) analyses were subsequently applied to calculate sensitivity at predefined $90 \%$ specificity in comparison to respective matched healthy controls. The statistical software $R$ (version 2.14.2 (2012-02-29)) [http://www.r-project.org] was used for all analyses.

\section{Results}

Candidate autoantibody identification in patients with RA Comparison of autoreactivity to 5892 antigens in sera from 72 patients with established RA (cohort A) and 116 therapynaive patients with early RA (cohort B) resulted in an intersect of 23 antigens with an individual $p$ value $<0.05$ and fold change $>1$ (Fig. 1). Fold changes ranged from 1.1 to 2.7 as detailed in Table 1.

\section{Candidate autoantibody identification in seronegative patients with RA}

In order to more specifically assess autoantibodies in seronegative RA, intersects of significantly increased autoantibodies in the seronegative subgroup of cohort B (termed B-) and an independent therapy-naïve cohort of 184 seronegative patients with RA (cohort C) was sought, resulting in 13 antigenic targets. Fold changes ranged from 1.1 to 1.8 as outlined in Table 2 .

\section{Candidate autoantibody identification in all cohorts}

Significantly increased autoantibodies in all cohorts were then identified resulting in diverse intersects as shown in Fig. 1. Significantly increased autoantibodies to three antigens were noted in all three cohorts (A, B and $C$ ) with fold changes ranging from 1.1 to 1.5 as detailed in Table 3. 


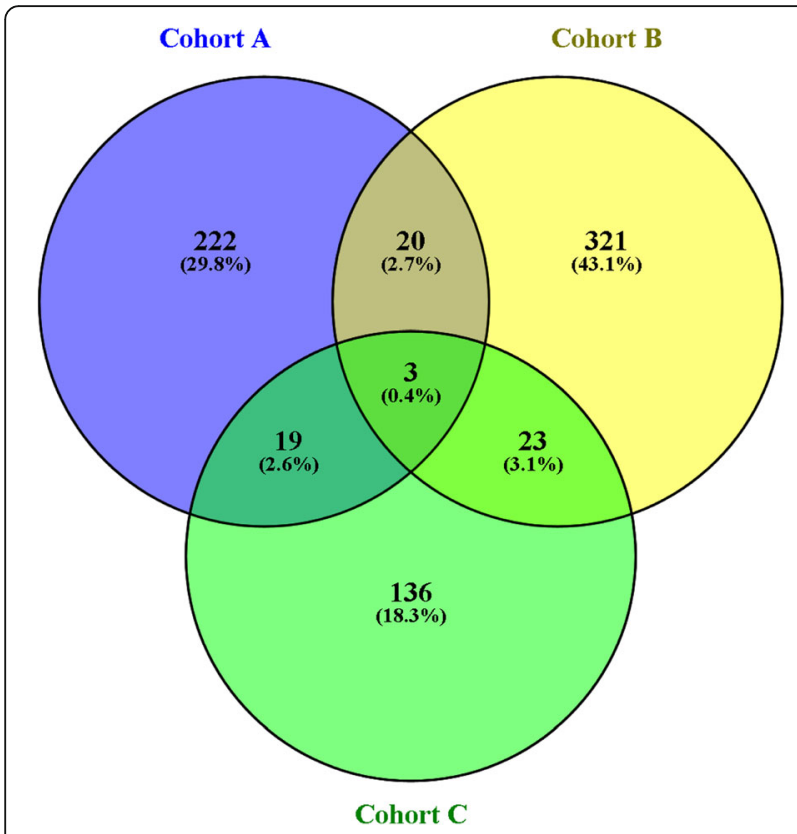

Fig. 1 Intersects of significantly increased lgG-autoantibodies

\section{Discussion}

In the current study, we set out to identify new diagnostic markers in RA with the use of an extensive screening for IgG autoantibodies. The strength of this study consists in the comprehensive inclusion of a vast number of potential autoantigens and the subsequent narrowing of the candidate list, employing independent patient cohorts. Importantly, cohorts $\mathrm{B}$ and $\mathrm{C}$ represented sera from therapy-naïve patients with early $\mathrm{RA}$, emphasizing the potential significance of our results for early serological diagnosis of the disease. Moreover, parts of the analyses focused on seronegative patients, resulting in identification of new interesting targets.

The risk of over-fitting the data is inherent in the adopted discovery approach with consideration of over 5800 antigens. Larger patient numbers were restricted due to practicability and cost considerations. Thus, we concentrated on single antigen performance and had to refrain from testing marker combinations. Furthermore, correction for multiple testing would have rendered the discovery approach unpromising a priori. Rather than direct diagnostic implications, the reported antigens are therefore considered attractive candidates, subject to improved ELISA-based assay development and subsequent clinical testing. The advantages of improved laboratory diagnosis are evident, because $30 \%$ of the RA patient population cannot be identified applying diagnostic testing for ACPA and/or rheumatoid factor (RF) [8].

We and others assume that a certain percentage of seronegative patients are truly seronegative, i.e. do not generate autoantibodies in the course of the disease, whereas other patients have autoantibodies against so far undisclosed antigens [13]. We further expect that this group of false-negative seronegative patients is heterogeneous, and that detection of low-frequency autoantibodies is required for serological detection. Only unmodified antigens were tested and only IgG antibodies were considered in the current study. Modifications such as citrullination and carbamylation [14] carry the potential for better detection rates and may yet unmask a greater proportion of seronegative patients with RA. However, we chose to use non-modified proteins (i.e. without citrullination, carbamylation or acetylation) as we follow the hypothesis that RA may have several different pathophysiological routes, of which the formation of autoantibodies against post-translational modifications and protein complexes is just one (major) route. We find support for this hypothesis in the previous discovery of non-modified antigens such as RA33 (HNRNPA2/B1) and 14-3-3 $[15,16]$. Interestingly, $14-3-3$ is an antigen both in unmodified and in citrullinated form [17].

Similarly, we found vimentin in its native form (as confirmed by tandem mass spectrometry) to be an autoantigen in both cohorts A and B [18], and vimentin is known to be a major target of ACPA in its citrullinated form [19]. We further assumed that novel autoantibodies would most likely be present in smaller subsets of patients. Low prevalence is a common feature of autoantibodies in other autoimmune diseases: in systemic sclerosis several antigens have been described, which occur with a prevalence between 5 and $10 \%$ (Th/T0, U3RNP, PM/Scl) [20], and in systemic lupus over 100 autoantibodies have been published, many of which have a prevalence below $15 \%$ [21]. This makes their discovery difficult, because the ratio between high numbers of antigens used in omicstype screening is already in imbalance to the number of available patient samples. This imbalance is further complicated if novel markers are present in only $5-15 \%$ of cases. Of note, in the present study, sensitivity of antigens across all group comparisons at a predefined specificity of $90 \%$ was $17.7 \pm 7.5 \%$, ranging from 2 to $41 \%$. Even though the study was designed to identify targets rather than determine their individual diagnostic performance, these results seem promising for individual diagnostic assay development. Furthermore, future validation of these diagnostic candidates should be carried out, ideally in early RA and include disease controls such as psoriatic arthritis.

Even though we used both established RA (cohorts A) and early RA (cohort B and C) for marker identification with the aim of identifying ubiquitous antigens in RA, the use of more homogeneous cohorts (e.g. seronegative patients only, or patients with early RA only) would represent an alternative approach to antigenic target identification. Clearly, the population used for screening 
Table 1 Intersect of significantly increased lgG-autoantibodies in patients with established RA (cohort A) and early RA (cohort B)

\begin{tabular}{|c|c|c|c|c|c|c|c|c|}
\hline \multicolumn{2}{|l|}{ Antigen } & \multirow[b]{2}{*}{ Gene ID } & \multicolumn{3}{|c|}{ Cohort A } & \multicolumn{3}{|c|}{ Cohort B } \\
\hline symbol & Name & & $P$ & FC & S & $P$ & FC & $\mathrm{S}$ \\
\hline DCTN1 & Dynactin 1 & 1639 & 0.026 & 1.5 & 14 & 0.001 & 1.5 & 19 \\
\hline GNPTG & $\mathrm{N}$-acetylglucosamine-1-phosphate transferase & 84572 & 0.021 & 1.2 & 17 & 0.001 & 1.3 & 13 \\
\hline HNRNPA1 & Heterogeneous nuclear ribonucleoprotein A1-like 2 & 144983 & 0.020 & 1.3 & 22 & 0.000 & 1.3 & 34 \\
\hline ITFG3 & Integrin alpha FG-GAP repeat containing 3 & 83986 & 0.048 & 1.1 & 19 & 0.014 & 1.1 & 23 \\
\hline APOA4 & Apolipoprotein A-IV & 337 & 0.025 & 1.1 & 35 & 0.001 & 1.2 & 11 \\
\hline CCDC136 & Coiled-coil domain containing 136 & 64753 & 0.039 & 2.2 & 14 & 0.031 & 1.7 & 2 \\
\hline CKAP4 & Cytoskeleton-associated protein 4 & 10970 & 0.027 & 1.2 & 22 & 0.000 & 1.3 & 28 \\
\hline CLCN2 & Chloride channel 2 & 1181 & 0.037 & 1.3 & 19 & 0.016 & 1.2 & 10 \\
\hline DMTF1 & Cyclin D binding myb-like transcription factor 1 & 9988 & 0.047 & 1.3 & 22 & 0.022 & 1.3 & 11 \\
\hline FAM59B & Family with sequence similarity 59 . member B & 150946 & 0.047 & 1.9 & 18 & 0.000 & 1.5 & 15 \\
\hline IGFBP2 & Insulin-like growth factor binding protein & 3485 & 0.015 & 1.2 & 19 & 0.000 & 1.5 & 13 \\
\hline TMCO7 & Transmembrane and coiled-coil domains 7 & 79613 & 0.009 & 1.1 & 21 & 0.000 & 1.5 & 20 \\
\hline USP48 & Ubiquitin specific peptidase 48 & 84196 & 0.001 & 2.7 & 18 & 0.034 & 1.7 & 11 \\
\hline VIM & Vimentin & 7431 & 0.022 & 1.3 & 19 & 0.000 & 1.7 & 38 \\
\hline ZFAND2B & Zinc finger. AN1-type domain 2B & 130617 & 0.034 & 1.2 & 18 & 0.002 & 1.3 & 22 \\
\hline ATP6V1A & ATPase. $\mathrm{H}+$ transporting. lysosomal $70 \mathrm{kDa}$. V1 subunit A & 523 & 0.048 & 1.2 & 21 & 0.042 & 1.3 & 11 \\
\hline GSN & Gelsolin & 2934 & 0.008 & 1.4 & 26 & 0.001 & 1.4 & 12 \\
\hline GSPT2 & G1 to $S$ phase transition 2 & 23708 & 0.045 & 1.2 & 14 & 0.043 & 1.3 & 8 \\
\hline HSBP1 & Heat shock factor binding protein 1 & 3281 & 0.021 & 1.2 & 25 & 0.002 & 1.3 & 19 \\
\hline NONO & Non-POU domain containing. octamer-binding & 4841 & 0.010 & 1.4 & 21 & 0.016 & 1.3 & 17 \\
\hline PRAP1 & Proline-rich acidic protein 1 & 118471 & 0.030 & 1.3 & 14 & 0.035 & 1.6 & 9 \\
\hline YES1 & YES proto-oncogene 1. Src family tyrosine kinase & 6714 & 0.032 & 1.1 & 28 & 0.010 & 1.2 & 13 \\
\hline SSB & Sjogren syndrome antigen B (autoantigen La) & 6741 & 0.009 & 1.4 & 15 & 0.042 & 1.1 & 17 \\
\hline
\end{tabular}

Cohort A: established rheumatoid arthritis (RA) $(n=72)$. Cohort B: early RA $(n=116) . P$ is $p$ value for fold change (FC) and sensitivity $(S)$ at $90 \%$ specificity compared to matched healthy controls, according to the Mann-Whitney test

approaches largely influences the resulting candidates. Thus, we maintain that an antibody identification strategy by an omics-type approach should be regarded as a means to reduce the number of potential individual targets. This in turn enables targeted individual autoantibody validation studies. We found support for this approach in other screening studies which similarly resulted in, albeit different, sets of potential targets [13]. This is probably due to different patient populations in terms of numbers of patients and ethnicity, and differences in the autoantibody identification approach [13], amongst other reasons. Nevertheless, all screening approaches result in potential targets for further individual testing and carry the potential for future diagnostic improvements, as has elegantly been demonstrated previously $[13,22]$.

After comprehensive screening of over 5800 antigens, we describe 23 potential new diagnostic antigens in RA and 13 for seronegative, early RA. As both currently used diagnostic antibodies (e.g. RF and ACPA antibodies) are involved in the pathogenesis of RA [4], it is interesting to speculate which functionalities the newly identified antigenic targets may have, as this might open up new fields of research into the pathogenesis of RA.

Some of the antigens found have previously been linked to RA. Of the three antigens identified in all groups, increased levels of insulin-like growth factor binding protein 2 (IGFBP2) were found in sera from patients with RA, and these correlate with CRP levels and were speculated to contribute to catabolic states in RA [23]. Heterogeneous nuclear ribonucleoprotein A1 (HNRNPA1) has previously been associated with RA, systemic lupus erythematosus and mixed connective tissue diseases and other rheumatic diseases [15]. A clear connection between $\mathrm{N}$-acetylglucosamine-1-phosphate transferase (GNPTG) and RA is so far unrecognized.

Of the antigens identified specifically in seronegative cohorts, a pathophysiological connection to actin beta (ACTB) is conclusive: autoreactive citrullinated epitopes of beta actin have been identified in synovial fluid in RA [24]. Moreover, pharmacological inhibition of actin cytoskeleton dynamics influences the pathogenicity of fibroblasts in arthritis [25]. A testable hypothesis would 
Table 2 Intersect of significantly increased lgG-autoantibodies in independent cohorts of patients with seronegative RA (cohorts B- and C)

\begin{tabular}{|c|c|c|c|c|c|c|c|c|}
\hline \multicolumn{3}{|l|}{ Antigen } & \multicolumn{3}{|c|}{ Cohort B- } & \multicolumn{3}{|c|}{ Cohort C } \\
\hline Symbol & Name & Gene ID & $P$ & FC & S & $P$ & $\mathrm{FC}$ & $S$ \\
\hline GNPTG & $\mathrm{N}$-acetylglucosamine-1-phosphate transferase. gamma subunit & 84572 & 0.001 & 1.4 & 16 & 0.04 & 1.1 & 10 \\
\hline HNRNPA1 & Heterogeneous nuclear ribonucleoprotein A1-like 2 & 144983 & 0.000 & 1.4 & 41 & 0.000 & 1.1 & 24 \\
\hline ACTB & Actin beta & 60 & 0.004 & 1.1 & 20 & 0.000 & 1.2 & 27 \\
\hline HSPD1 & Heat shock 60 kDa protein 1 (chaperonin) & 3329 & 0.008 & 1.6 & 14 & 0.002 & 1.8 & 17 \\
\hline PER1 & Period homolog 1 & 5187 & 0.000 & 2.2 & 24 & 0.001 & 1.5 & 13 \\
\hline SMARCD3 & $\begin{array}{l}\text { SWI/SNF related. matrix associated. actin dependent regulator } \\
\text { of Chromatin. subfamily } d \text {. member } 3\end{array}$ & 6604 & 0.016 & 1.3 & 14 & 0.016 & 1.1 & 14 \\
\hline SMYD2 & SET and MYND domain containing 2 & 56950 & 0.007 & 1.6 & 10 & 0.05 & 1.4 & 5 \\
\hline IGFBP2 & Insulin-like growth factor binding protein 2. $36 \mathrm{kDa}$ & 3485 & 0.003 & 1.5 & 16 & 0.03 & 1.1 & 14 \\
\hline KLKB1 & Kallikrein B. plasma (Fletcher factor) 1 & 3818 & 0.03 & 1.2 & 10 & 0.03 & 1.2 & 13 \\
\hline GON4L & Gon-4-like (C. elegans) & 54856 & 0.01 & 1.2 & 12 & 0.02 & 1.1 & 15 \\
\hline CHMP5 & Chromatin modifying protein 5 & 51510 & 0.03 & 1.5 & 12 & 0.004 & 1.3 & 14 \\
\hline DHX15 & DEAH (Asp-Glu-Ala-His) box polypeptide 15 & 1665 & 0.007 & 1.5 & 12 & 0.03 & 1.1 & 11 \\
\hline FAHD2A & Fumarylacetoacetate hydrolase domain containing $2 \mathrm{~A}$ & 51011 & 0.04 & 1.2 & 16 & 0.05 & 1.2 & 14 \\
\hline
\end{tabular}

Cohort B-: seronegative rheumatoid arthritis $(\mathrm{RA})(\mathrm{n}=51)$. Cohort C: patients with seronegative RA $(\mathrm{n}=184)$. $P$ is $p$ value for fold change $(\mathrm{FC})$ and sensitivity $(\mathrm{S})$ at $90 \%$ specificity compared to matched healthy controls, according to the Mann-Whitney test

thus be that autoantibodies to beta actin might alter the cytoskeleton assembly and functionality of synovial fibroblasts towards an RA phenotype. Further, plasma kallikrein (antibodies to the beta subunit (KLKB1) were identified) is part of a cascade that results in the activation of bradykinins, which are considered to be potent inflammatory mediators with relevance in arthritis [26].

Of the intersecting antigens identified in the mixed cohorts A and B, gelsolin (GSN) was recently identified as a promising urinary biomarker for RA [27]. Increased levels of apolipoprotein A4 (APOA4) have been identified in sera from patients with RA using a proteomic approach [28]. Decreased levels of proline-rich acidic proteins have been identified in patients with RA reporting oral sicca symptoms [29]. The roles of the respective antibodies, like PRAP1-antibodies, have not been investigated in this regard so far. Moreover, dynactin 1 (DCTN1) has been shown to be an integral part of osteoclast formation and function [30]. Finally, even though antibodies to SSB are a hallmark of Sjögren's syndrome, these antibodies are also found in RA [31].
The identified antibodies to these known targets deserve further functional studies to determine their potential role in the pathogenesis of RA. Of note, most of the antigenic targets identified have not been thoroughly investigated in the context of RA and may thus open up new fields of research. Future studies will adopt the challenge of largescale screening for post-translationally modified antigens, and to assess the individual performance of the retrieved candidates.

\section{Conclusions}

Comprehensive sequential autoantibody profiling revealed novel, highly interesting, IgG-autoantibodies for future diagnostic assay development and pathophysiological research, especially in seronegative RA.

\section{Abbreviations}

ACPA: anti-citrullinated peptide antibodies; ACR: American College of Rheumatology; ACTB: actin beta; APOA4: apolipoprotein A4; BBA: bead-based assay; BSA: bovine serum albumin; DAS28: Disease Activity Score for 28 joints; ELISA: enzyme-linked immunosorbent assay; EULAR: European League Against Rheumatism; GNPTG: N-acetylglucosamine-1-phosphate transferase; GSN: gelsolin; HNRNP: heterogeneous nuclear ribonucleoprotein;

Table 3 Intersect of significantly increased lgG autoantibodies in patients with RA (cohorts A, B, and C)

\begin{tabular}{|c|c|c|c|c|c|c|c|c|c|c|c|}
\hline \multicolumn{3}{|l|}{ Antigen } & \multicolumn{3}{|c|}{ Cohort A } & \multicolumn{3}{|c|}{ Cohort B } & \multicolumn{3}{|c|}{ Cohort C } \\
\hline symbol & Name & Gene ID & P & FC & S & P & FC & S & $P$ & FC & S \\
\hline GNPTG & $\mathrm{N}$-acetylglucosamine-1-phosphate transferase & 84572 & 0.021 & 1.2 & 17 & 0.001 & 1.3 & 13 & 0.04 & 1.1 & 10 \\
\hline HNRNPA1 & Heterogeneous nuclear ribonucleoprotein A1-like 2 & 144983 & 0.020 & 1.3 & 22 & 0.000 & 1.3 & 34 & 0.001 & 1.1 & 24 \\
\hline IGFBP2 & Insulin-like growth factor binding protein 2 & 3485 & 0.01 & 1.2 & 19 & 0.000 & 1.5 & 13 & 0.03 & 1.1 & 15 \\
\hline
\end{tabular}

Cohort A: patients with established rheumatoid arthritis (RA) $(n=72)$. cohort B: early RA $(n=116)$. cohort C: early seronegative RA ( $=184)$. $P$ is $p$ value for fold change (FC) and sensitivity (S) at $90 \%$ specificity compared to matched healthy controls, according to the Mann-Whitney test 
IGFBP: insulin-like growth factor binding protein; KLK: plasma kallikrein; MFI: mean fluorescence activity; PBS: phosphate-buffered saline; PRAP: proline-rich acidic proteins; RA: rheumatoid arthritis; RF: rheumatoid factor; RT: room temperature; TNF: tumor necrosis factor

\section{Acknowledgements}

The authors are grateful for the technical assistance of Petra Rengers for the processing of serum samples, Martin Gamer for antigen production and Daniel Chamrad, Laura Schlieker and Klaus Marquart for assistance with data analysis.

\section{Funding}

SV, RB, RFB, JR, EB and MS gratefully acknowledge financial support from the Hiller Foundation. Antigen production was in part funded by grant CLIB2021, 0316041A. Parts of the study (HIT HARD) were supported by the German Ministry of Education and Science (BMBF).

\section{Availability of supporting data}

Not applicable.

\section{Authors' contributions}

SV conceived the study, generated clinical data, analyzed data, interpreted data and drafted the manuscript. AL conceived the study, generated biomarker data, analyzed data and interpreted data. PB generated biomarker data and analyzed data. HDZ performed statistical analysis and analyzed data. HG generated biomarker data and analyzed data. RB participated in statistical analysis, data analysis and data interpretation. RFB, JR, EB, JD, H-EL, AS and G-RB generated clinical data and interpreted data. PSK and MS conceived the study, analyzed and interpreted data and drafted the manuscript. All authors read, critically revised and approved the final version of the manuscript.

\section{Authors' information}

Not applicable.

\section{Competing interests}

AL, PB, HDZ, HG and PSK are employees of Protagen AG, Germany. The other authors declare that they have no competing interests.

\section{Consent for publication}

Not applicable.

\section{Ethical approval and consent to participate}

Informed patient consent was obtained from all patients and the study was approved by the Ethics Committee of the Medical Faculty of Heinrich Heine University Düsseldorf (study number 2850) and by the Ethics Committee of the Bavarian Medical Chamber (Bayrische Ärztekammer, study number 01/09).

\section{Author details}

'Department Rheumatology \& Hiller Research Unit Rheumatology, Heinrich-Heine-University, Moorenstr. 5, Düsseldorf 40225, Germany. ${ }^{2}$ Protagen AG, Dortmund, Germany. ${ }^{3}$ German Diabetes Center, Institute for Biometry and Epidemiology, Düsseldorf, Germany. ${ }^{4}$ Department Rheumatology and Clinical Immunology, Charité - University Medicine Berlin, Berlin, Germany. ${ }^{5}$ Rheumatology, Clinical Immunology and Osteololgy at Evangelisches Krankenhaus, Düsseldorf, Germany.

Received: 1 April 2016 Accepted: 20 September 2016 Published online: 12 October 2016

\section{References}

1. Mclnnes IB, Schett $G$. The pathogenesis of rheumatoid arthritis. N Engl J Med. 2011:365:2205-19.

2. Vander Cruyssen B, Peene I, Cantaert T, Hoffman IEA, De Rycke L, Veys EM, et al. Anti-citrullinated protein/peptide antibodies (ACPA) in rheumatoid arthritis: specificity and relation with rheumatoid factor. Autoimmun Rev. 2005;4:468-74.

3. Charpin C, Arnoux F, Martin M, Toussirot E, Lambert N, Balandraud N, et al. New autoantibodies in early rheumatoid arthritis. Arthritis Res Ther. 2013;15:R78.

4. Harre U, Georgess D, Bang H, Bozec A, Axmann R, Ossipova E, et al. Induction of osteoclastogenesis and bone loss by human autoantibodies against citrullinated vimentin. J Clin Invest. 2012;122:1791-802.
5. Kleyer A, Finzel S, Rech J, Manger B, Krieter M, Faustini F, Araujo E, Hueber AJ, Harre U, Engelke K, Schett G. Bone loss before the clinical onset of rheumatoid arthritis in subjects with anticitrullinated protein antibodies. Ann Rheum Dis. 2014;73(5):854-60.

6. van der Helm-van AHM, Verpoort KN, Breedveld FC, Toes REM, Huizinga TWJ. Antibodies to citrullinated proteins and differences in clinical progression of rheumatoid arthritis. Arthritis Res Ther. 2005;7:R949-958.

7. Ursum J, Bos WH, van Dillen N, Dijkmans BA, van Schaardenburg D. Levels of anticitrullinated protein antibodies and IgM rheumatoid factor are not associated with outcome in early arthritis patients: a cohort study. Arthritis Res Ther. 2010;12:R8.

8. Detert J, Bastian H, Listing J, Weiß A, Wassenberg S, Liebhaber A, et al. Induction therapy with adalimumab plus methotrexate for 24 weeks followed by methotrexate monotherapy up to week 48 versus methotrexate therapy alone for DMARD-naive patients with early rheumatoid arthritis: HIT HARD, an investigator-initiated study. Ann Rheum Dis. 2013;72:844-50.

9. Büssow K, Cahill D, Nietfeld W, Bancroft D, Scherzinger $E$, Lehrach $H$, et al. A method for global protein expression and antibody screening on highdensity filters of an arrayed cDNA library. Nucleic Acids Res. 1998;26:5007-8.

10. Rual J-F, Hirozane-Kishikawa T, Hao T, Bertin N, Li S, Dricot A, et al. Human ORFeome version 1.1: a platform for reverse proteomics. Genome Res. 2004;14:2128-35.

11. Brinkmann U, Mattes RE, Buckel P. High-level expression of recombinant genes in Escherichia coli is dependent on the availability of the dnaY gene product. Gene. 1989;85:109-14

12. Bolstad BM, Irizarry RA, Astrand M, Speed TP. A comparison of normalization methods for high density oligonucleotide array data based on variance and bias. Bioinforma Oxf Engl. 2003;19:185-93.

13. Somers K, Geusens P, Elewaut D, De Keyser F, Rummens J-L, Coenen M, et al. Novel autoantibody markers for early and seronegative rheumatoid arthritis. J Autoimmun. 2011;36:33-46.

14. Shi J, Knevel R, Suwannalai P, van der Linden MP, Janssen GMC, van Veelen PA et al. Autoantibodies recognizing carbamylated proteins are present in sera of patients with rheumatoid arthritis and predict joint damage. Proc Natl Acad Sci USA. 2011;108:17372-7.

15. Steiner $G$, Skriner $K$, Smolen JS. Autoantibodies to the A/B proteins of the heterogeneous nuclear ribonucleoprotein complex: novel tools for the diagnosis of rheumatic diseases. Int Arch Allergy Immunol. 1996;111:314-9.

16. Maksymowych WP, Marotta A. 14-3-3n: a novel biomarker platform for rheumatoid arthritis. Clin Exp Rheumatol. 2014;32:S-35-39.

17. Maksymowych A, Bykerk V, van der Heijde D, Landewé R, Murphy M, Marotta A. SAT0036 Auto-Antibodies to pan and citrullinated 14-3-3 ETA are expressed in anti-CCP negative RA. Ann Rheum Dis. 2013;72 Suppl 3:A592.

18. Alcover A, Ramirez-Lafita F, Hernandez C, Nieto A, Avila J. Antibodies to vimentin intermediate filaments in sera from patients with SLE and RA: quantitation by solid phase radioimmunoassay. J Rheumatol. 1985;12:233-6.

19. Vossenaar ER, Després N, Lapointe E, van der Heijden A, Lora M, Senshu T, et al. Rheumatoid arthritis specific anti-Sa antibodies target citrullinated vimentin. Arthritis Res Ther. 2004;6:R142-150.

20. Walker JG, Fritzler MJ. Update on autoantibodies in systemic sclerosis. Curr Opin Rheumatol. 2007;19:580-91.

21. Sherer $Y$, Gorstein A, Fritzler MJ, Shoenfeld Y. Autoantibody explosion in systemic lupus erythematosus: more than 100 different antibodies found in SLE patients. Semin Arthritis Rheum. 2004:34:501-37.

22. De Winter LM, Hansen WLJ, van Steenbergen HW, Geusens P, Lenaerts J, Somers $\mathrm{K}$, et al. Autoantibodies to two novel peptides in seronegative and early rheumatoid arthritis. Rheumatology. 2016;55:1431-6.

23. Neidel J. Changes in systemic levels of insulin-like growth factors and their binding proteins in patients with rheumatoid arthritis. Clin Exp Rheumatol. 2001;19:81-4.

24. van Beers JJBC, Schwarte CM, Stammen-Vogelzangs J, Oosterink E, Božič B, Pruijn GJM. The rheumatoid arthritis synovial fluid citrullinome reveals novel citrullinated epitopes in apolipoprotein $\mathrm{E}$, myeloid nuclear differentiation antigen, and $\beta$-actin. Arthritis Rheum. 2013;65:69-80.

25. Vasilopoulos Y, Gkretsi V, Armaka M, Aidinis V, Kollias G. Actin cytoskeleton dynamics linked to synovial fibroblast activation as a novel pathogenic principle in TNF-driven arthritis. Ann Rheum Dis. 2007:66(3):iii23-28.

26. Cassim B, Mody G, Bhoola K. Kallikrein cascade and cytokines in inflamed joints. Pharmacol Ther. 2002;94:1-34.

27. Stalmach A, Johnsson H, Mclnnes IB, Husi H, Klein J, Dakna M, et al. Identification of urinary peptide biomarkers associated with rheumatoid arthritis. PLoS One. 2014;9:e104625. 
28. Li T, Zheng B, Huang Z, Lin Q, Zhao L, Liao Z, et al. Screening diseaseassociated proteins from sera of patients with rheumatoid arthritis: a comparative proteomic study. Chin Med J (Engl). 2010;123:537-43.

29. Jensen JL, Uhlig T, Kvien TK, Axéll T. Characteristics of rheumatoid arthritis patients with self-reported sicca symptoms: evaluation of medical, salivary and oral parameters. Oral Dis. 1997;3:254-61.

30. Ng Py C, Zhao H, Ye S, Sm Ang E, Khor E, Feng H, et al. Disruption of the dynein-dynactin compex unveils motor-specific functions in osteoclast formation and bone resorption. J Bone Min Res. 2013;28:119-34.

31. Manoussakis MN, Kistis CG, Aidinis V, Guialis A, Piha L, Sekeris CE, et al. Detection of human-specific anti-la(SSB) antibodies in patients with rheumatoid arthritis. J Autoimmun. 1995;8:959-69.

Submit your next manuscript to BioMed Central and we will help you at every step:

- We accept pre-submission inquiries

- Our selector tool helps you to find the most relevant journal

- We provide round the clock customer support

- Convenient online submission

- Thorough peer review

- Inclusion in PubMed and all major indexing services

- Maximum visibility for your research

Submit your manuscript at www.biomedcentral.com/submit
Biomed Central 